\section{$\underset{\substack{\text { hommes } \\ \text { \& migrations }}}{ }$}

\section{Hommes \& migrations}

Revue française de référence sur les dynamiques

migratoires

$1320 \mid 2018$

Au prisme de la consommation

\title{
La construction sociale de « l'en commun » par la consommation
}

Les sociétés réunionnaise et malaisienne

\section{Laurence Tibère}

\section{(2) OpenEdition}

Journals

Édition électronique

URL : http://journals.openedition.org/hommesmigrations/4044

DOI : 10.4000/hommesmigrations.4044

ISSN : 2262-3353

Éditeur

Musée national de l'histoire de l'immigration

Édition imprimée

Date de publication : 1 janvier 2018

Pagination : 31-39

ISBN : 978-2-919040-40-7

ISSN : 1142-852X

Référence électronique

Laurence Tibère, «La construction sociale de «l'en commun » par la consommation », Hommes \& migrations [En ligne], 1320 | 2018, mis en ligne le 01 janvier 2021, consulté le 06 janvier 2021. URL : http://journals.openedition.org/hommesmigrations/4044; DOI : https://doi.org/10.4000/ hommesmigrations.4044 


\section{LA CONSTRUCTION SOCIALE DE « L'EN COMMUN » PAR LA CONSOMMATION : LES SOCIÉTÉS RÉUNIONNAISE ET MALAISIENNE}

Par LAURENCE TIBÈRE, maître de conférences en sociologie, Certop-Cnrs, université de Toulouse-2 (France), Chair of Food Studies, université de Taylors (Malaisie).

Au sein de sociétés multiculturelles comme La Réunion ou la Malaisie, I'alimentation joue un rôle central dans la construction d'une identité collective fondée sur les migrations multiples. L'histoire de la cuisine créole réunionnaise, comme celle des repas entre communautés en Malaisie en sont deux exemples frappants. Le partage de mets, de plats, de recettes, et la commensalité faisant de chacun un compagnon de tablée contribuent en effet, le temps d'un repas, au dialogue des cultures. Ainsi, la consommation alimentaire offre-t-elle un espace de construction de l'en commun tout en laissant s'exprimer les différences.

La Réunion et la Malaisie sont deux sociétés multiculturelles issues des migrations. Elles constituent des terrains très riches pour étudier les processus sociaux ou politiques qui sous-tendent la construction et le maintien, au sein des sociétés, d'espaces abstraits et/ou concrets de rencontre et de partage, entre les différentes composantes. C'est ce que j'appelle « l'en commun ». Il y a plusieurs années, j'ai étudié l'alimentation à La Réunion en cherchant à comprendre la façon dont la créolisation se manifestait concrètement dans la (les) cuisine(s) et plus largement, l'alimentation, mais aussi dans les imaginaires collectifs ${ }^{1}$. J'ai pu y saisir le rôle des nourritures dans la construction à la fois des représentations collectives et des évènements sociaux concrets, qui sous-tendent lélaboration des identités collectives ${ }^{2}$. Par la suite, j’ai étudié une autre société structurée autour des migrations : la Malaisie. Je me suis intéressée là encore aux imaginaires sociaux sur certaines composantes alimentaires (boissons, ingrédients, plats, cuisines...), qui font dire aux gens en quelque sorte: "C'est notre bien commun et ça nous représente - au-delà de nos différences. » Sur ce point, j'ai analysé les discours des mangeurs mais aussi ceux d'acteurs institutionnels intervenant dans lélaboration de ces composantes emblématiques. J'ai aussi étudié la place de ces composantes dans la vie concrète des populations, dans les prises alimentaires quotidiennes ou festives. 
Sur le plan théorique, la réflexion s'adossait à trois principales approches. Celles de Claude Lévi-Strauss et de Mary Douglas sur le statut symbolique des nourritures, et celles proposées par Claude Fischler sur la fonction incorporatrice de l'alimentation ${ }^{3}$. Et un peu plus tard, celles sur les repas également, sur leur rôle structurant du social et des identités collectives ${ }^{4}$. Mon questionnement rejoignait plus largement ceux de la sociologie générale sur ce qui fait société, autour à la fois de ce qui « rattache à l'être social dans sa totalité » en tant qu'entité collective et ceux «interindividuels » inscrits dans l'interaction ${ }^{5}$. Ont également été utiles les analyses relatives au fonctionnement des sociétés culturellement diversifiées et aux dynamiques par lesquelles, au-delà des différences, des distances voire des tensions, elles construisent aussi de l'en commun ${ }^{6}$. Je propose ici de faire état des analyses menées à $\mathrm{La}$ Réunion et en Malaisie, deux contextes qui différent par leur histoire et les modalités des recompositions migratoires en leur sein, mais dans lesquels on repère des espaces, imaginaires mais aussi concrets, de partage interculturel autour de l'alimentation.

\section{La cuisine créole : un modèle alimentaire construit avec les migrations}

La société réunionnaise s'est formée avec les migrations. À l'arrivée des premiers colons français en 1663, l'île était inhabitée. Au fil de la colonisation, des populations venues d'Europe, d'Inde, d'Afrique, de Madagascar, de Chine, s'y sont installées et ont créé une société nouvelle. On peut retenir trois

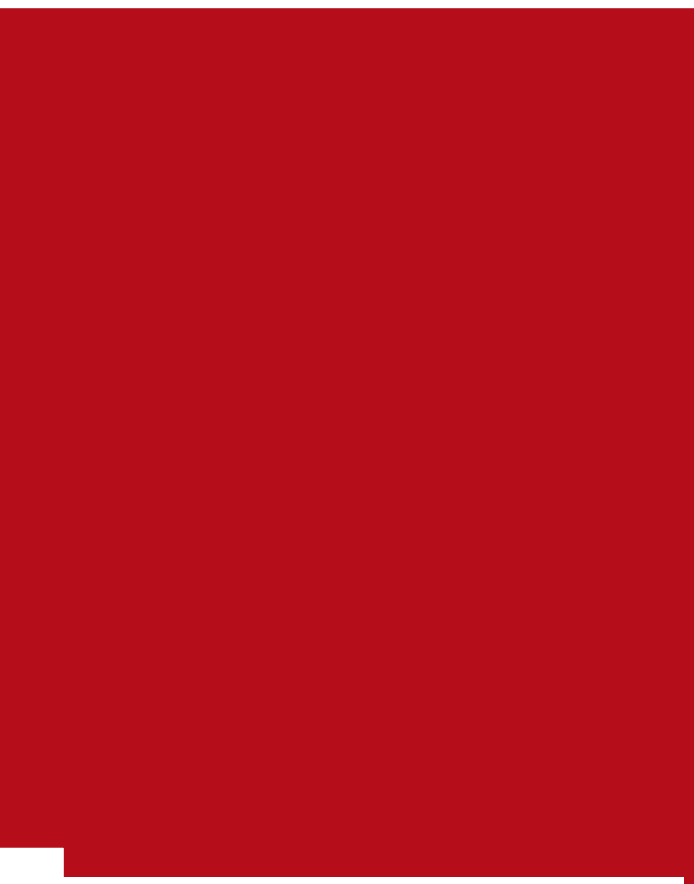

Cuisine créole réunionnaise, 2016. () LAURENCE TIBËRE.

grandes périodes migratoires : une première vague qui va du XVII (au début de la colonisation) à la moitié du XIX siècle (après l'abolition de l'esclavage). C'est durant ces premiers temps que la cuisine créole s'élabore autour des influences culturelles européennes, indo-portugaises, malgaches, africaines, à partir de ce qui est amené des pays d'origine et de ce qui est disponible sur l'île. Une seconde période commence après 1848, avec l'arrivée de populations engagées notamment pour remplacer la main-d'œuvre servile et venant en particulier d'Inde (Tamouls issus du Tamil Nadu et musulmans du Gujerat) et de Chine (Cantonnais et Hakkas issus du Nord de la Chine). Ils vont progressivement influencer le système culinaire créole existant (au niveau des préparations, des plats, des épices, des ingrédients, des ustensiles...) et vont

3. Claude Lévi-Strauss, The origin of table manners, New York, Harper \& Row, 1968 ; Mary Douglas, Implicit Meanings: Selected Essays in Anthropology, Londres, Routledge, 1975 ; Claude Fischler, L'Homnivore, Paris, Odile Jacob, 1990. 4. Émile Durkheim, The Elementary Forms of the Religious Life: A Study in Religious Sociology, Londres, Allen \& Unwin, 1912 ; Nils-Arvid Bringéus, Man, Food and Milieu: A Swedish Approach to Food Ethnology, Édimbourg, Tuckwell Press, 2001 ; Jean-Pierre Poulain, Sociologies de l'alimentation, Paris, PUF, 2017. 5. Salvador Juan, Formes élémentaires de la vie quotidienne, Paris, PUF, 1995, citant Émile Durkheim, "La science positive de la morale en Allemagne ", in Revue philosophique, $n^{\circ}$ 24, 1887, pp. 33-58. 6. Benedict Anderson, Imagined communities, New York, Verso, 1991; Charles Taylor, Multiculturalism: Examining the Politics of Recognition, Princeton, N.J, Princeton University Press, 1994 ; Andréas Semprini, Le multiculturalisme, Paris, Presses universitaires de France, 2000 ; Will Kymlicka, Multicultural Citizenship: A Liberal Theory of Minority Rights, Oxford, Oxford University Press, 1995. 
eux-mêmes en adopter de nombreuses composantes, tout en gardant des spécificités de leurs propres systèmes culturels (repas de fêtes, interdits religieux...). Cette mise en commun est en grande partie rendue possible selon la période, grâce aux interactions de voisinage, mais aussi aux unions mixtes, aux relations de travail, à la présence des restaurants et les jeux d'appropriation et d'imitation, ou encore le travail des femmes de ménages cuisinières. Enfin, une troisième période migratoire, plus récente, concerne l'installation de populations principalement issues de France métropolitaine et, en moindre importance, de l'Océan indien? ${ }^{7}$ Mon but était de saisir, par l'alimentation, les dynamiques identitaires qui animent cette société et les relations réciproques qu'entretiennent les Réunionnais. Certains travaux existants m'ont guidée au départ. Ceux de Patrice Cohen, en particulier, posaient les contours de "l'espace social alimentaire réunionnais", avec ses formes et ses structures de repas, ses temporalités, son ordre du mangeable et ses variations d'un univers ethnoculturel à l'autre, les dégoûts, les interdits, les manières de table... en d'autres termes tout ce qui compose un modèle alimentaire ${ }^{8}$. Il montrait que cet ensemble était composé d’un espace appelé localement "cuisine créole " et d'espaces (bien que fortement créolisés) adossés aux cuisines de référence des diverses composantes de la population réunionnaise (les univers ethnoculturels des Cafres, Yabs, Zarabs, Malbars, et Chinois) ${ }^{9}$.

\section{Un espace de contribution}

Lors des entretiens, les Réunionnais eux-mêmes évoquaient spontanément l'existence de cette cuisine créole qu'ils percevaient comme commune, à côté d'autres plus spécifiques, plus différentielles. L'autre aspect récurrent dans les propos était que cette cuisine créole résultait des apports des différentes composantes ethnoculturelles réunionnaises. Une première dimension importante de l'imaginaire collectif autour de cette cuisine se dégageait ici : celle de la contribution. Le témoignage suivant illustre bien cette représentation d'un territoire commun que chaque groupe a influencé. Un interviewé rencontré dans sa cuisine me montre son pilon ${ }^{10}$ et me dit : "Tu vois le pilon? [et il me montre chaque coin] Ça c'est l'influence française et européenne, ça c'est l'influence africaine et malgache, ça c'est l'Inde et ça c'est la Chine... tout ça s'est mélangé ici [il fait le geste de piler avec le pilon] pour donner la cuisine créole. » (Homme, 42 ans, métis Cafre et Malbar ${ }^{11}$.) J'avais aussi relevé, dans un livre sur les Réunionnais musulmans de la commune de SaintPierre, ces propos : "Le Réunionnais d'origine gujerati (originaire du Gujerat) n'a-t-il pas apporté à l'île une part de sa culture? Le samoussa est devenu un mets national réunionnais ${ }^{12}$. " Les Chinois font également ce genre de démonstrations dans les entretiens, à propos des bouchons ou du siaw ${ }^{13}$ : «Les bouchons, par exemple, disons que ça fait partie de la

7. Entre 2002 et 2005, j'ai réalisé 43 entretiens et observations approfondies. J'ai aussi mené une enquête par questionnaire auprès de 545 personnes (échantillon représentatif sur les variables "sexe ", "âge ", " microrégions " et CSP). Je n'ai pas étudié, dans l'enquête qualitative, les représentations des populations plus récentes, issues de Métropole (les Zoreys), de Mayotte ou de Madagascar. En revanche, elles ont été prises en compte dans l'enquête quantitative sur les normes et les pratiques alimentaires. Les détails de la méthodologie et des résultats sont présentés dans le livre : Laurence Tibère, L'alimentation dans le "vivre-ensemble " multiculturel, op. cit. 8. Patrice Cohen, Le cari partagé. Anthropologie de l'alimentation à lîle de la Réunion, Paris, Karthala, 200o. 9. Les Cafres désignent les Réunionnais noirs; les Yabs correspondent aux Réunionnais blancs issus de familles modestes; les Zarabs sont les Réunionnais originaires du Gujerat, musulmans; les Malbars sont pour la plupart des Indiens originaires du Tamil Nadu ; les Chinois (ou Sinois) désignent les Réunionnais originaires de Chine. À ce que je qualifie « d’univers ethnoculturels » cafre, chinois, malbar, yab, zarabe, zoréole sont attachés, au-delà des seuls éléments "visibles », des systèmes de valeurs, des héritages réels ou imaginaires, des attitudes. À La Réunion, la complexité réside dans l'absence possible de coïncidence entre les caractères identitaires extérieurs comme la couleur de peau et la subjectivité à partir de laquelle l'individu s'inscrit dans tel ou tel univers. Un individu dont le phénotype correspond au Blanc peut s'identifier comme Cafrine (Noir). Sur le plan religieux également, un même individu peut revendiquer deux appartenances. 10. Le pilon est un mortier en roche volcanique présent dans toutes les cuisines à La Réunion. Il sert à la préparation des aromates et épices. 11. Les univers ethnoculturels sont repris tels que les expriment spontanément les interviewés. 12. Amode Ismael-Daoudjee, Les Indo-musulmans Gujaratis (Zarabes) et La Mosquée-Médersa de Saint-Pierre de La Réunion, La Saline, La Réunion, GRAHTER (Groupe de recherche sur l'archéologie et I'histoire de la terre réunionnaise), 2002, p. 8. 13. Les bouchons sont des bouchées cuites à la vapeur. Le siaw est le nom sino-réunionnais pour la sauce de soja. 
interne, au sein de la société réunionnaise, le terme « créole » désigne les Blancs, les Noirs et les métis, soit les populations issues des premières vagues de migrations. Mais, quand il s'agit de différencier les Réunionnais en général des gens qui viennent de l'extérieur, le terme « créole » désigne tous les Réunionnais ${ }^{15}$. Dans les démonstrations identitaires de ces populations sur l'appartenance à la société réunionnaise, la cuisine créole et, plus largement, le manger créole ont un statut symbolique particulier et les démonstrations spontanées autour du lien intégration-alimentation sont récurrentes. Dans les entretiens, lorsqu'ils parlent de leur histoire, de celle de leur famille, la cuisine créole apparaît très souvent, avec la langue et l'attachement à l'île, comme une preuve de leur intégration. J'en donnerai quelques exemples : « Je suis intégrée ici... Je mange créole, c'est la cuisine qu'on mange chez nous... disons... tous les jours. " (Femme, 45 ans, Zarabe.) Ou encore ce témoignage que je trouve cuisine créole maintenant. " (Homme, 34 ans, exemplaire : "On n'est pas des gens à part, la preuve, la Chinois réunionnais.) Dans l'imaginaire collectif, la cuisine créole avec sa structure riz/grain/cari/ piment, etc. ${ }^{14}$, avec ses plats et ses préparations emblématiques, est un patrimoine commun. Elle est aussi un marqueur d'intégration...

\section{Et une preuve d'intégration}

J'ai accordé une attention particulière aux perceptions des Réunionnais originaires d'Inde et de Chine, arrivés plus tardivement, après la formation de la société créole initiale, celle des premiers temps du peuplement. Différents travaux sur la créolité en tant que marqueur local d'intégration montraient qu'en cuisine et tout (...), on cuisine créole. » Ce type de démonstration est aussi mobilisé pour prouver la créolité de l'autre... Je me souviens d'une femme créole blanche qui, à propos de son mari d'origine chinoise, déclare : «Il mange du shop suey ${ }^{16}$, mais avec des grains et du rougay [éléments emblématiques de la cuisine créole]. Alors, il est chinois, mais il est aussi créole. » Certains parents d'enfants issus d'unions entre Métropolitains et Réunionnais ( "Zoréoles », en créole) : "Il parle créole, il mange créole, le riz et le piment tout ça... c'est un vrai Créole. " (Femme, créole, mariée à un Zorey.) J'ai repris ces aspects dans l'enquête par questionnaire à travers la question suivante: «Donnez 3 critères qui vous font dire de quelqu'un qu'il est créole. " Le fait de manger la

14. La structure typique du repas créole s'organise autour du riz, sur lequel sont déposés les grains, le rougay, le cari et les brèdes (pousses végétales). Les grains sont les légumineuses, le cari est une préparation à base de tomate, de curcuma, d'ail et d'oignons préparée avec de la viande, du poisson ou des légumes. Le piment est servi frais ou préparé en rougay (préparation pimentée, associant le piment et les légumes ou les fruits notamment). 15. Prosper Eve, "Les péripéties d’une insertion : les indo-musulmans à la Réunion de la fin du $19^{e}$ siècle à 1939, relations historiques et culturelles entre France et Inde, XVII-XXe siècles ", in Actes de la conférence internationale France-Inde de I'AHIOI, Saint-Denis, 21-28 juillet 1986 ; Jean Benoist, "Héritage, naissance et structure d'une société ", in Actes du colloque La Réunion dans l'océan indien, Paris, La documentation française, 1985 ; Jean Benoist, Hindouismes créoles. Mascareignes, Antilles, Paris, CTHS, 1998 ; Robert Chaudenson, Les créoles français, Paris, Nathan, 1979 ; Des îles, des hommes, des langues. Langues créoles cultures créoles, Paris, L'Harmattan, 1992. 16. Sauté d'origine chinoise, constitué de légumes et de viande (ou de produits de la mer). 
cuisine créole arrive en seconde position après la langue ${ }^{17}$. J'ajouterai qu'à l'époque de l'enquête cette incorporation n'était pas seulement imaginée, puisque les repas créoles étaient consommés le midi $(43,1 \%)$ et le soir $\left(43,4 \%{ }^{18}\right)$.

De tout cela, je concluais que la cuisine créole était une ressource identitaire pour les Réunionnais. Elle s'était construite comme telle au fil des migrations et des inter-influences portées par la créolisation. En Malaisie, ce sont d'autres processus qui portent l'en commun. À côté des dynamiques populaires, d'autres sont repérables, développées dans le cadre de la gestion politique de la multiculturalité ${ }^{19}$.

\section{One Malaysia ! La société multiculturelle malaisienne}

Ancienne colonie britannique devenue indépendante en 1957, la société malaisienne est composée de quatre groupes ethniques officiels au sein d'une population de 31 millions d'habitants ${ }^{20}$ : les populations indigènes (Aborigènes, Orang Asli...) et les Malais (implantés entre le XIV et le XV ${ }^{\text {e }}$ siècle et convertis à l'islam), ces derniers constituant aujourd'hui le groupe dominant. Ces deux composantes forment les Bumiputras (en malais, « fils du

17. Laurence Tibère, "Manger créole... ", op. cit. ; L'alimentation dans le "vivre ensemble " multiculturel..., op. cit. 18. Recueil des pratiques effectives réalisé à partir du rappel des consommations des dernières 24 heures. 19. Will Kymlicka, Baogang He (dir.), Multiculturalism in Asia, Oxford, Oxford University Press, 2005. Je mobiliserai des données qualitatives et quantitatives issues de l'enquête nationale sur l'alimentation des Malaisiens réalisée entre 2012 et 2013 à laquelle j'ai participé. La collecte qualitative s'organisait autour de 32 entretiens semi-directifs réalisés à Kuala Lumpur ainsi que d’un inventaire des supports papiers (journaux, magazines) et télévisuels en lien avec le patrimoine alimentaire ; l'enquête par questionnaires portait sur un échantillon de 2000 individus, représentatif de la population nationale. Voir Jean-Pierre Poulain, Wendy Smith, Cyrille Laporte, Laurence Tibère, Noor Mohd Ismail, Elise Mognard, Marcella Aloysius, Ari Ragavan Neethiahnanthan, Amri Baharuddin Shamsul, "Studying the consequences of modernization on ethnic food patterns: Development of the malaysian food barometer (Mfb) ", in Anthropology of Food, avril 2015. 20. À titre comparatif, la population réunionnaise compte 850000 habitants. 
sol », considérés comme ayant une antériorité sur le territoire). Viennent ensuite, par ordre d'importance numérique, les Chinois et les Indiens, installés en Malaisie depuis plusieurs générations ${ }^{21}$. Les migrants chinois et indiens ont adopté progressivement le malais pour langue, tout en conservant leurs langues d'origine. Malgré une organisation cloisonnée et le maintien au sein des différents groupes de nombreux traits culturels spécifiques, des transformations culturelles ont eu lieu du fait des emprunts et des adaptations, sans pour autant que se déploient des mouvements globaux de mise en commun et de valorisation des métissages tels que ceux opérés dans la créolisation ${ }^{22}$.

Pour Éric Olmedo, en effet, les

Dans l'alimentation, les

différents univers culturels

se sont transformés eux aussi

pour donner des versions

malaisiennes des cuisines

de référence. Et certaines

préparations, certains plats,

initialement consommés

par l'une des composantes

de la société malaisienne,

ont été adoptés et adaptés

par les autres. processus anthropologiques de création d'une nouvelle société et d'une nouvelle culture à partir des influences portées par les différentes populations migrantes, n'ont pas eu lieu en Malaisie, du fait notamment de l'antériorité de certaines populations ${ }^{23}$. Shamsul Amri Baharuddin précise que le métissage, même s'il existe, n'est pas revendiqué comme une donnée constitutive de l'identité collective malaisienne. Il précise que la culture «Nonya », fruit des métissages entre les Chinois et les Portugais qui ont eu lieu au XV'e siècle à Malacca, ne représente qu'une tendance très minoritaire aujourd'hui ${ }^{24}$. La culture commune et la cohésion sociale résultent, selon lui, des formes d'interactions qui ont lieu dans la vie quotidienne entre les Malaisiens. Enfin, à propos des métis, Lim souligne le fait qu'ils sont dévalorisés et cela encore plus avec les cloisonnements ethniques contemporains ${ }^{25}$.

Dans l'alimentation, les différents univers culturels se sont transformés eux aussi pour donner des versions malaisiennes des cuisines de référence. Et certaines préparations, certains plats, initialement consommés par l'une des composantes de la société malaisienne, ont été adoptés et adaptés par les autres. Le Nasi Lemak, plat consommé à l'origine par les travailleurs malais de la côte ouest de la péninsule, est de ceux-là ${ }^{26}$. En malais, il signifie littéralement «riz gras » ou «riz dans la crème » ${ }^{27}$. Il est servi dans une assiette ou une feuille de bananier pliée en pyramide, pour l'emporter. Le Nasi Lemak apparaît comme l'un des plats - sinon le plat national - dans tous les dépliants à destination des visiteurs.

\section{Un emblème de «l'unité dans la diversité »}

Dans un dossier du magazine de la Malaysia Air Line distribué dans les avions, le Nasi Lemak figure parmi les "plats dont les Malaisiens devraient être fiers ${ }^{28}$ ". Un concours culinaire annuel mettant en compétition les vendeurs de Nasi Lemak a été créé il y a quelques années. L'engouement est également fort dans les médias télévisés où, en 2002 déjà, la chaîne de télévision Fried Chillie Food Network (du groupe national Unify Hypp TV), dans une émission intitulée "Malaysian eat. That's what we do ", ouvrait son 
dossier spécial Nasi Lemak en soulignant ses qualités non seulement nutritionnelles, mais aussi gustatives et pratiques, qui en feraient un plat consommé tout au long de la journée, et cela, par toutes les composantes de la société malaisienne ${ }^{29}$. Ce dernier point, particulièrement important dans le contexte multiculturel malaisien, est très souvent développé par les médias locaux. Ainsi, une série malaisienne intitulée, Nasi Lemak 2.0, créée en 2011, est présentée lors de son lancement en ces termes par son réalisateur : «Le Nasi Lemak symbolise la cohésion nationale. » Et l'un des acteurs vedette de la série d'ajouter : «Le message que je veux faire passer est que le système en Malaisie est inégal... mais nous vivons ensemble, nous mangeons ensemble, il n'y a pas de racisme $e^{30}$. " La cohésion entre les différents groupes ethnoculturels qui composent la société malaisienne et les dynamiques politiques pour construire des symboles fédérateurs constituent, en effet, des enjeux majeurs. Depuis une dizaine d'années, la promotion par les instances politiques du slogan «One Malaysia », prônant « l'unité dans la différence », y fait écho. C'est dans ce contexte que se situe la candidature de certaines composantes de la cuisine malaisienne, parmi lesquelles le Nasi Lemak figure en tête, au patrimoine culturel immatériel de l'Unesco $^{31}$. Le consensus institutionnel et médiatique autour de ce plat met en lumière le rôle des institutions, et en particulier des pouvoirs publics, dans la construction d'emblèmes nationaux.

Dans les sociétés multiculturelles, les enjeux autour de la cohésion nationale et de la régulation des relations entre les différentes composantes culturelles sont particulièrement importants et différents auteurs ont montré le rôle central qu'y tiennent les symboles fédérateurs ${ }^{32}$. En Malaisie, comme dans de nombreuses sociétés du sud-est asiatique où la pluralité ethnique et religieuse est devenue la norme, la gestion politique de la diversité est centrale ${ }^{33}$. Cette gestion institutionnelle cohabite la plupart du temps avec la gestion populaire ou «vernacular » selon le terme de Beng Huat Chua ${ }^{34}$. Qu'en est-il du statut du Nasi Lemak? Quelle place occupe-t-il dans la vie sociale des Malaisiens ? Nous verrons que sa consommation met en lumière l'importance d'un autre espace de partage et de rencontres autour de l'alimentation, celui des repas et de la commensalité entre les différents groupes ethniques.

\section{La commensalité interethnique autour du Nasi Lemak}

Soulignons, tout d'abord, que l'importance symbolique du Nasi Lemak, même si elle est instrumentalisée par les instances politiques, est présente dans les représentations collectives. En effet, les entretiens réalisés avec les Malaisiens confirment leur attachement à ce plat, l'un d'eux déclarant même : "La vie est impossible en Malaisie sans le Nasi Lemak. »(Homme, 32 ans, Indien-Malaisien.) Deux qualités lui sont systématiquement associées : l'adaptabilité et l'accessibilité. Sur le premier plan, il est présenté comme facile à cuisiner et, aspect important, à agrémenter selon les goûts et les moyens. Des ajustements s'opèrent ainsi selon l'univers ethnoculturel, les Indiens étant réputés cuisiner un sambal (condiment à base de piment)

29. Le message original est le suivant : "You know... we should make Nasi Lemak as our national dish. Not only can you get all your protein, carbohydrates, fiber and vitamins in one dish, different places will give you different types of chili kicks and swings depending on the sambal they serve. The Malaysian Nasi Lemak has also changed style in the last ten years or so. Now, instead of being just a breakfast meal, it can now be enjoyed throughout the day and night and accepted by all the races in Malaysia. Totally unique!". 30. "My message is that the system in Malaysia is unequal... But when we live together, we eat together, there is no racism " (Fred Chong, Wee Meng Chee, interview AFP, septembre 2011). 31. Lors d'un entretien, les représentants du National Heritage Board en charge du dossier pointaient les enjeux politiques de la présence du Nasi Lemak en première position à côté d'autres plats représentatifs de chacune des composantes ethniques comme le Roti Canaï et certains plats chinois (Entretien réalisé en août 2015 à Kuala Lumpur). 32. Charles Taylor, Multiculturalism: Examining the Politics of Recognition, Princeton, N.J Princeton University Press, 1994 ; Andréas Semprini, Le multiculturalisme, Paris, Presses universitaires de France, 2000 ; Will Kymlicka, op. cit. 33. Nadesan Ganesan, "Liberal and Structural Ethnic Political Accommodation in Malaysia ", in Will Kymlicka, Baogang He (dir.), op. cit. ; Éric Olmedo, op. cit. 34. Beng Huat Chua, "Modernism and the vernacular: transformation of public spaces and social life in Singapore", in Journal of Architectural and Planning Research, vol. 8, n 3, 1991, pp. 203-221. 
plus épicé, tandis que les Malais ajoutent du lait de coco et du sucre à la préparation et les Chinois valorisent la saveur salée $e^{35}$. L'autre dimension récurrente dans les propos est la possibilité d'adapter le Nasi Lemak en fonction des interdits religieux (sans porc pour les musulmans, ou sans bœuf pour les Hindous, végétariens pour certains...). Il est aussi question, dans les discours, de l'accessibilité physique du Nasi Lemak que l'on trouve dans tous les lieux de restauration.

\section{L'importance}

du Nasi Lemak ne tient

peut-être pas - ou plus -

tant à sa consommation

massive au quotidien, qu'à la commensalité interethnique, hors foyer, dont il fait l'objet dans le cadre de repas amicaux ou entre collègues, notamment.

Dans une étude des espaces publics de consommation et les modalités selon lesquelles la multiculturalité et les interactions entre les différentes composantes ethniques se déploient en Malaisie et à Singapour, Jean Duruz et Gaïk Cheng Khoo montrent que tous les Malaisiens, quelles que soient leurs origines sociales et ethniques, fréquentent certains lieux de restauration populaires ${ }^{36}$. Ils précisent que les politiques de discrimination raciale promue par la NEP (New Economic Policy) et l'islamisation de la société malaisienne ont entraîné la baisse de la commensalité interethnique au foyer, en particulier entre Chinois et Malais, beaucoup plus courante dans les années 1960 et $1970^{37}$. Ils ajoutent, cependant, que celleci reste active dans la restauration hors foyer, et soulignent le statut moteur de l'univers professionnel et de l'école sur ce plan. Je me suis, de ce fait, intéressée aux contextes de consommation du Nasi Lemak et, en particulier, à la commensalité interethnique dont il faisait éventuellement l'objet. En termes de consommation effective, le Nasi Lemak est présent principalement le matin, dans $12,4 \%$ des petits déjeuners - ce qui est significatif si l'on tient compte de l'hyper choix qui existe en Malaisie mais révèle une tendance à sa survalorisation, tant dans les discours institutionnels que populaires. $\mathrm{Ce}$ qui me paraît intéressant, cependant, c'est qu'il est consommé à l'extérieur dans $42 \%$ des cas, et $45 \%$ dans le cadre d'une commensalité entre les différentes composantes ethniques. L'importance du Nasi Lemak ne tient peut-être pas - ou plus - tant à sa consommation massive au quotidien, quà la commensalité interethnique, hors foyer, dont il fait l'objet dans le cadre de repas amicaux ou entre collègues, notamment. Cela rappelle l'importance des repas pris ensemble et souligne leur statut particulier dans la construction de l'en commun.

\section{D'autres espaces populaires de commensalité}

Dans Les formes élémentaires de la vie religieuse, Émile Durkheim soulignait le caractère fédérateur du repas partagé et le fait qu'il crée entre ceux qui y assistent « un lien de parenté artificielle $e^{38}$ ». Il ajoute que la répétition de rituels confère aux participants un sentiment fort de connexion les uns aux autres. Mary Douglas et Baron Isherwood soulignent eux aussi le fait que le repas est un " processus rituel qui donne du sens au flux des événements ${ }^{39}$ ».

J'ai observé en Malaisie d'autres éléments relatifs au repas qui me semblent intervenir dans le maintien du lien social interethnique. Le premier concerne les food-court, espaces de restauration hors foyer organisés autour d'une offre multi-ethnique. Les Malaisiens s'y retrouvent pour partager un repas autour d'une table, chacun pouvant choisir selon ses préférences et ses interdits alimentaires. L'autre élément concerne les Open House. Il s'agit de repas organisés à certains moments des calendriers religieux durant lesquels les Malaisiens des autres ethnies et univers religieux sont conviés à domicile.

35. Michael Hsiao Hsin-Huang, Lim Khay-Thiong, "History and politics of national cuisine : Malaysia and Taiwan ", in Kwang Ok Kim (dir.), Re-orienting Cuisine: East Asian Foodways in the Twenty-First Century, New York/Oxford, Berghahn Books, 2015, pp. 31-55.

36. Jean Duruz, Gaïk Cheng Khoo, Eating Together: Food, Space, and Identity in Malaysia and Singapore, Lanham, Rowman \& Littlefield Publishers, 2014. 37. Concernant les grandes lignes de la NEP (Nouvelle politique économique) instaurée dans les années 1970, nous invitons le lecteur à lire l'ouvrage de Edmund T. Gomez, Johan Saravanamuttu, The New Economic Policy in Malaysia. Affirmative Actions, Ethnics Inequalities and Social Justice, Singapour, NUS Press, 2012. 38. Émile Durkheim, op. cit., p. 481. 39. Mary Douglas, Baron Isherwood, The World of Goods, Londres, Routledge, 1979. 
Ainsi, les Malais invitent des Malaisiens indiens ou chinois au moment de Hari Raya (fin du Ramadan). Les Chinois et les Indiens chrétiens font de même au moment des fêtes de Noël. Les Hindous le font à l'occasion du Dipavali (fête de la lumière). Les Bouddhistes le font pour le Nouvel An, etc. Les repas, souvent organisés autour de buffets, respectent les interdits religieux des convives (halal, absence de bœuf, etc.). Ces moments festifs sont, semble-t-il, très importants dans la cohésion sociale. Et, si j'ai repéré à La Réunion l'existence d'une commensalité inter-ethnique, elle est moins institutionnalisée. En fait, à La Réunion, les relations entre les composantes se font aussi au sein même des familles avec les mariages mixtes. En Malaisie, où la société est plus cloisonnée, les repas interethniques prennent d'autres formes et ont un statut symbolique très fort. Une interviewée me disait au sujet des Open Houses : "Quand tu es invité, tu ne peux pas dire non (...). Ça ne se fait pas et, même si tu as 4 invitations dans la même journée, et bien, tu $y$ vas et tu restes le temps que tu peux. Tout le monde comprendra. " (Femme, 37 ans, ChinoiseMalaisienne.) Ses propos soulignent le statut d'institution sociale de ces repas et les engagements sociaux réciproques que génèrent les invitations qui les encadrent.

\section{Conclusion}

J'ai voulu saisir le rôle des consommations alimentaires dans deux sociétés fortement structurées par les migrations. À La Réunion, des populations ont été mises en contact sur une île déserte, se sont culturellement influencées pour fabriquer, à partir des ressources existantes et de leurs cultures de référence, des sociétés et des cultures nouvelles. La cuisine créole fait partie de ces créations originales et a fonctionné comme une matrice qui a absorbé les influences au fil des migrations. Les Réunionnais se représentent cette cuisine comme un bien commun, fruit de la contribution collective. En Malaisie, où la gestion politique de la multiculturalité est pratiquée, le Nasi Lemak est largement mobilisé par les instances politiques et médiatiques pour symboliser «l'unité dans la diversité ». À côté de ces dynamiques politiques, des espaces populaires de commensalité se déploient dans certains lieux de consommation, tels que les food courts et au rythme d'événements festifs comme les Open Houses. En Malaisie, comme à La Réunion, des populations avec leurs histoires entremêlées ont été amenées à cohabiter. Elles ont fait de la cuisine et de la consommation alimentaire la matière pour construire des espaces de rencontre avec, il est vrai, des différences liées aux conditions d'existence au sein de la société coloniale, à son histoire, aux modalités politiques de régulation de la diversité. Bien entendu, la cuisine et l'univers alimentaire portent aussi des espaces de différenciation et de distanciation sociale et culturelle, mais ce qui m'intéresse c'est la façon dont, malgré ces aspects, chaque société invente des dispositifs servant de supports à l'en commun. Il me semble que l'alimentation joue un rôle non négligeable dans ces processus. Dans le contexte métropolitain, il serait intéressant d'étudier le statut de l'alimentation dans cette perspective, en lien avec les migrations passées et récentes. Repère-t-on des plats emblématiques? Des espaces de commensalité ? Sur ce dernier point, certains lieux de consommation tels que les Food Markets (cantines/marchés) jouent peut-être ce rôle de brassage social mais aussi culturel. 